\title{
Repeated Encephalopathy and Hemicerebral Atrophy in a Patient with Familial Hemiplegic Migraine Type 1
}

\author{
Yuichi Tashiro ${ }^{1}$, Tsuneo Yamazaki ${ }^{2}$, Shun Nagamine ${ }^{1}$, Yuji Mizuno ${ }^{1}$, \\ Adachi Yoshiki $^{3}$ and Koichi Okamoto ${ }^{1}$
}

\begin{abstract}
We herein describe a case of a 38-year-old man with familial hemiplegic migraine with a T666M mutation in the electrical potential-dependent calcium ion channel (CACNAIA) gene. His migraine was accompanied by hemiparesis and impaired consciousness. Brain magnetic resonance imaging revealed abnormalities in the right cortical hemisphere. Single-photon emission computed tomography demonstrated a decrease in iomazenil uptake and an increase in ${ }^{99 m}$ Tc-ethyl cysteinate dimer uptake at the ipsilateral site. Positron emission tomography showed a decrease in ${ }^{18} \mathrm{~F}$-fluorodeoxyglucose uptake in the same area, which later showed atrophic changes. The patient's brain atrophy ceased after treatment with sodium valproate. This case suggests that the progression of brain atrophy can be prevented with adequate prophylaxis.
\end{abstract}

Key words: familial hemiplegic migraine type 1, cerebral atrophy, encephalopathy, single-photon emission computed tomography (SPECT)

(Intern Med 53: 2245-2250, 2014)

(DOI: 10.2169/internalmedicine.53.0295)

\section{Introduction}

Familial hemiplegic migraine is an autosomal dominant hereditary migraine with aura. To date, three causative genes for this condition have been identified: CACNA1A (FHM1), ATP1A2 (FHM2), and SCN1A (FHM3) (1). Many missense mutation sites have been detected in each of these genes. Although some reports have detailed the clinical course of this disease complicated with cerebral atrophy, many cases with familial hemiplegic migraine are diagnosed solely by clinical criteria; therefore, they lack sufficient gene analysis data. We herein report a case of familial hemiplegic migraine due to a $\mathrm{T} 666 \mathrm{M}$ mutation in the electrical potentialdependent calcium ion channel (CACNAIA) gene for which repeated brain imaging was performed. During the course of the disease, magnetic resonance imaging (MRI) revealed hemispheric atrophy, and single-photon emission computed tomography (SPECT) demonstrated decreased ${ }^{123}$ I-iomazenil (IMZ) accumulation at the same site.

\section{Case Report}

A 38-year-old man with impaired consciousness was transferred to our hospital by ambulance. As a teenager, he repeatedly experienced eye twitching and was diagnosed with epilepsy by his family doctor. Sodium valproate was prescribed, but he discontinued regular treatment. At the age of 38 , the eye flicker recurred along with a high body temperature of $38^{\circ} \mathrm{C}$ and right limb numbness. All three symptoms vanished within several minutes. He consulted his doctor, and a brain MRI detected no abnormality. One month later, he lost consciousness after experiencing a high-grade fever and left hemiparesis. He was rediagnosed with epilepsy at another hospital and was admitted. A few days later, he regained consciousness and was discharged. Sodium valproate was prescribed; however, he did not take the medicine. The patient lost consciousness again the day after being discharged, and he was then transferred to our hospital where he presented in a semicomatose state with an absence of left limb movements. T2-weighted images and

\footnotetext{
${ }^{1}$ Department of Neurology, Gunma University Graduate School of Medicine, Japan, ${ }^{2}$ Gunma University Graduate School of Health Sciences, Japan and ${ }^{3}$ Matsue Medical Center, National Hospital Organization, Japan
}

Received for publication February 7, 2013; Accepted for publication March 23, 2014

Correspondence to Dr. Yuichi Tashiro, y.tashiro.d@mn.hosp.go.jp 

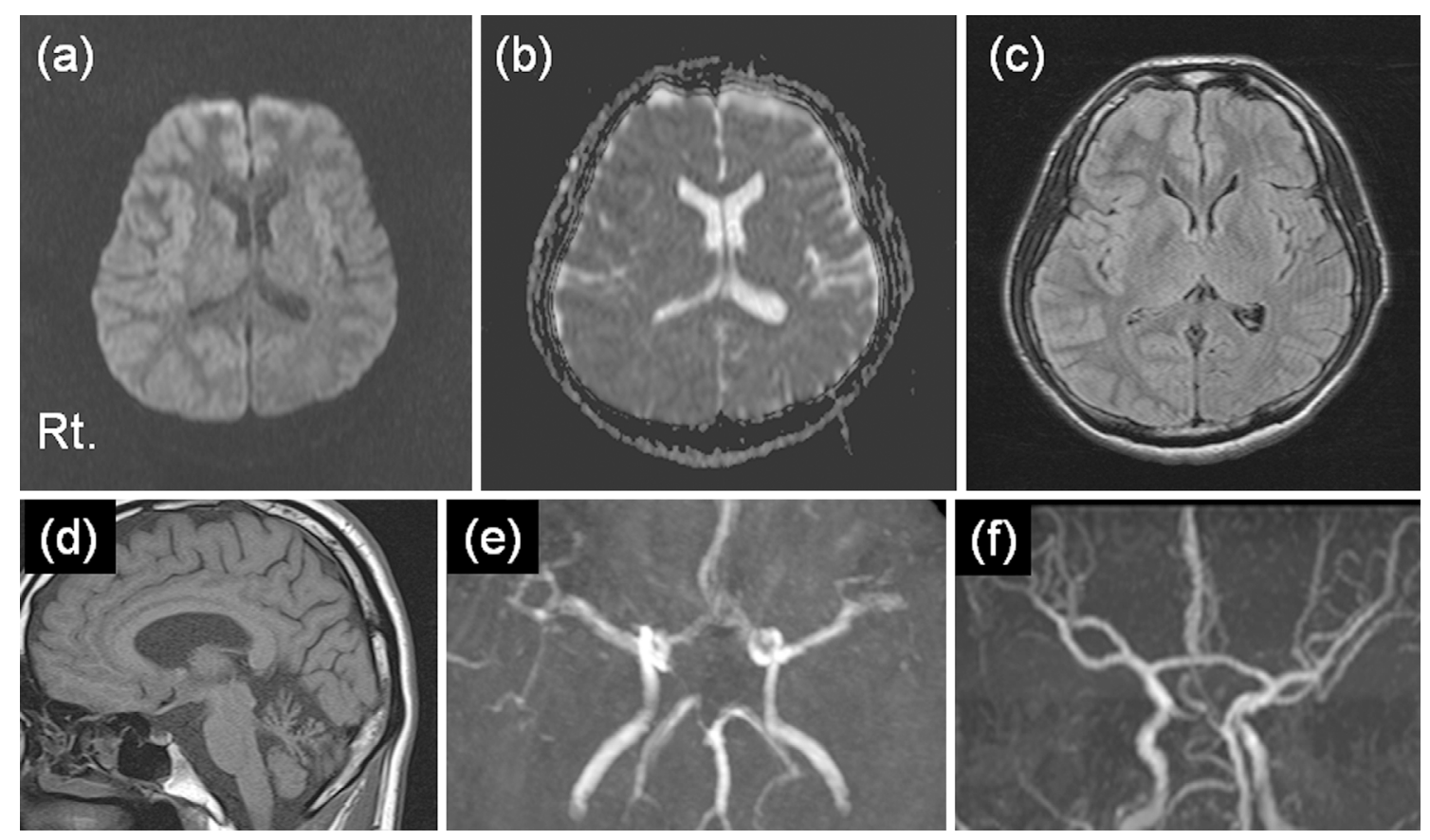

Figure 1. Brain MRI performed at the first admission. (a) DWI showing swelling in the right cortical hemisphere. (b) The apparent diffusion coefficient map, without a hypointense signal area. (c) FLAIR showing a slight hyperintense signal in the same area of the cortex. (d) A sagittal section of a T1-weighted image showing cerebellar atrophy. (e and f) Magnetic resonance angiography showing that the right middle cerebral artery was dilated in comparison with the left at the first hospital day (e) and recovered 15 days later (f). DWI: diffusion-weighted images, FLAIR: fluid-attenuated inversion recovery, MRI: magnetic resonance imaging

diffusion-weighted images (DWI) with brain MRI conducted at our facility revealed a hyperintensity in the right cortical hemisphere. Bilateral cerebellar atrophy was also evident. Magnetic resonance angiography showed mild right middle cerebral artery dilatation, suggesting increased blood flow in the area (Fig. 1). This speculation was confirmed by ${ }^{99 \mathrm{~m}} \mathrm{Tc}-$ ethyl cysteinate dimer (ECD) SPECT, which showed increased signaling in the right hemisphere (Fig. 2a). In contrast, IMZ-SPECT showed the accumulation of IMZ three days after administration (late imaging) to dramatically decrease in the ipsilateral site (Fig. 2b). ${ }^{18} \mathrm{~F}$-fluorodeoxyglucose (FDG) positron emission tomography (PET) demonstrated continuous decreased glucose metabolism in the right hemisphere (Fig. 2c), even 12 days after admission when the patient recovered consciousness. Electroencephalography revealed no epileptic discharge, but slow waves (theta waves) were evident in the right frontal and temporal lobes. His blood and cerebrospinal fluid analyses, including lactic acid and pyruvic acid readings, showed no abnormalities. A detailed history revealed that the patient had experienced several episodes of migraine with aura, sometimes combined with hemiparesis, since he was a teenager. His mother also reportedly experienced migraines with hemiparesis three times in her youth. Based on these facts, a clinical diagnosis of familial hemiplegic migraine was made without the aid of genetic analysis. The patient recovered completely and was discharged. He had no evident ataxia despite cerebellar atrophy. He was again prescribed sodium valproate, but discon- tinued taking it due to nausea. Four years later, he again suffered from migraine with aura, a high fever $\left(39.9^{\circ} \mathrm{C}\right)$, right hemiparesis, and loss of consciousness. He was readmitted to our hospital and recovered completely the next day. With his consent, a genetic analysis was performed, which revealed a $T 666 \mathrm{M}$ heteromer mutation in his CACNA1A gene exon 16 sequence (the antisense strand primer). The patient's mother later consulted us due to migraine problems and consented to undergo a genetic analysis of the CACNA1A gene, which confirmed that she possessed the same mutation. Sodium valproate was again prescribed, and he suffered no subsequent severe attacks, unconsciousness, or hemiparesis while taking the medication regularly. Fig. 3 shows the brain MRI [images with fluid-attenuated inversion recovery (FLAIR)] of a time-course study beginning with his first admission at our hospital to the present. Right hemispheric cerebral atrophy was initially evident, and the left hemisphere had mild atrophy; however, atrophy progression appeared to stop after his second admission, at least in the right hemisphere. Follow-up SPECT (Fig. 4) showed partially recovered levels of ECD and IMZ accumulation about four years after the first admission compared to the levels at the first admission. His regional cerebral blood flow calculated based on ECD accumulation is shown in Fig. 5. Hyperflow in the right cerebrum evident during his first hospital admission decreased about four years later. Since being discharged, the patient has led a normal life. His Mini-Mental State Examination score was 27. Follow-up 

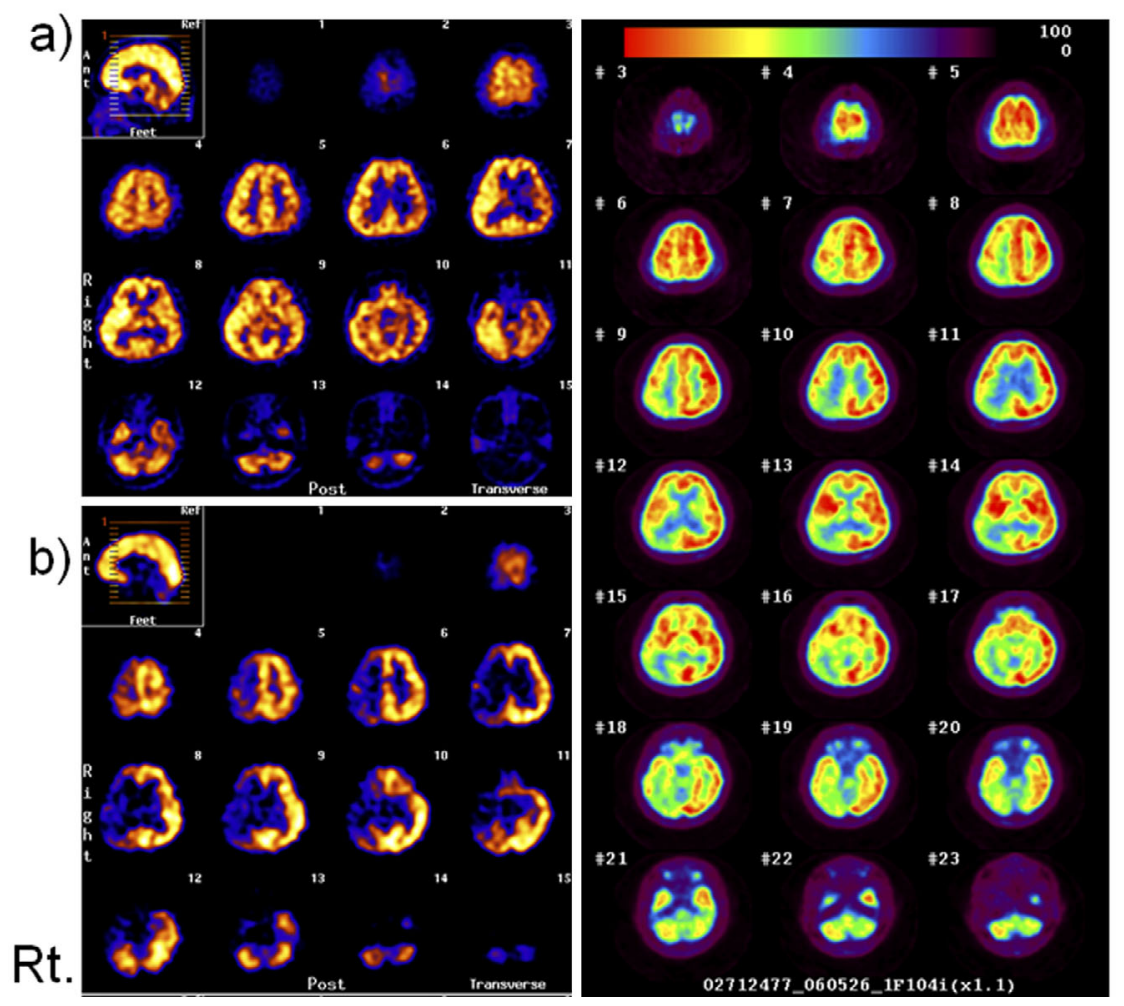

c)

Figure 2. (a) ECD-SPECT with an increase in ECD accumulation in the right cortical hemisphere (first admission, eighth hospital day). (b) IMZ-SPECT (first admission, third hospital day). (c) FDGPET (first admission, twelfth hospital day) showed decreased IMZ accumulation and FDG uptake in the same area. ECD-SPECT: ${ }^{99 \mathrm{~m}}$ Tc-ethyl cysteinate dimer single-photon emission computed tomography, IMZ-SPECT: ${ }^{123}$ I-iomazenil single-photon emission computed tomography, FDG-PET: ${ }^{18}$ F-fluorodeoxyglucose-positron emission tomography
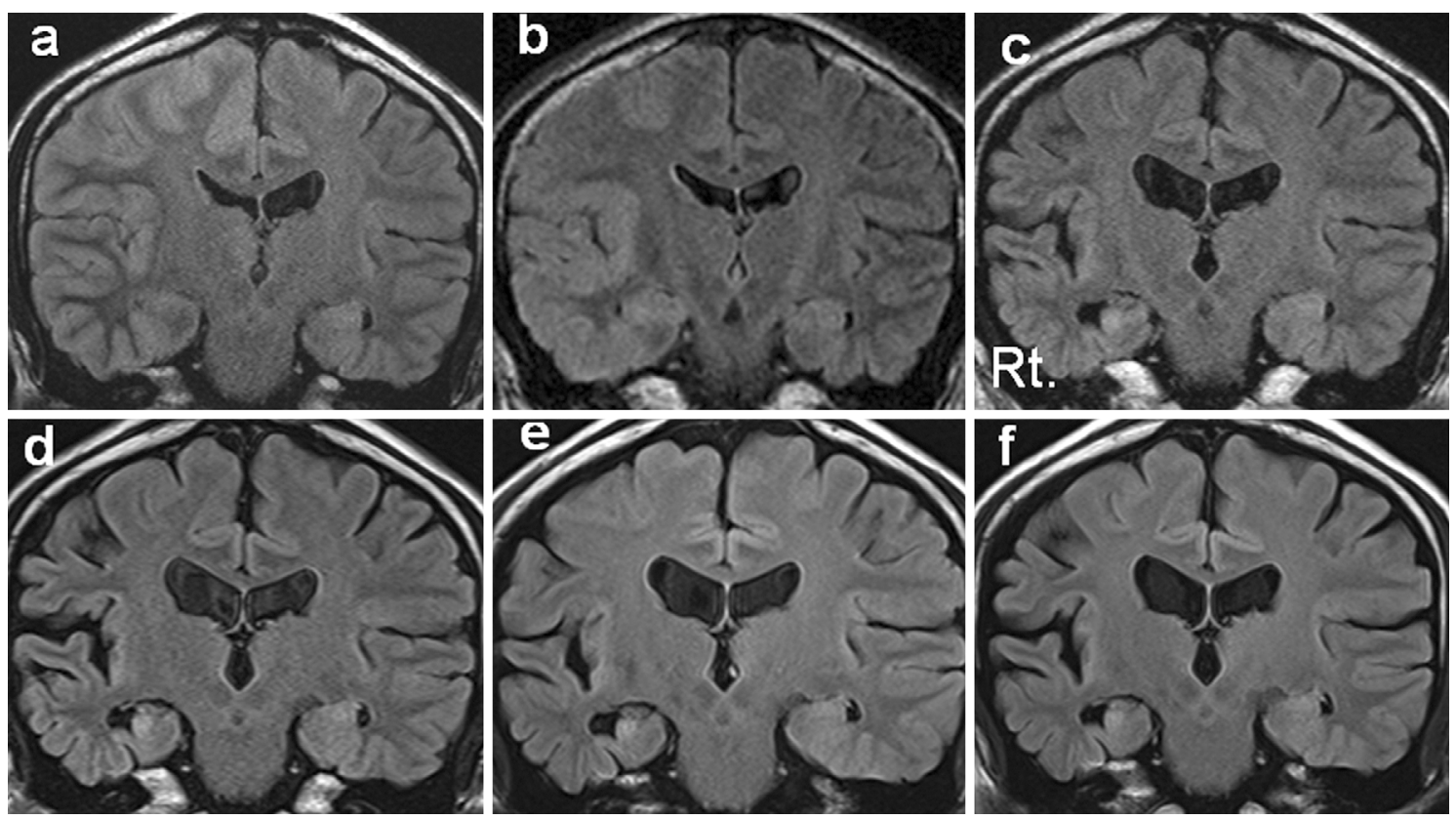

Figure 3. Brain MRI (FLAIR, coronal section) time-course study. (a and b) At first admission, right cortical hemisphere swelling and a hyperintense signal. (c and d) A follow-up study revealed atrophic changes in the ipsilateral hemisphere 12 months (c) and $\mathbf{4 4 . 5}$ months after (d) the first admission. (e) On the patient's second admission (45 months after the first admission), swelling was not obvious, but atrophy appeared to be the same in the right hemisphere. Left mild cortical atrophy was also demonstrated. (f) The patient's condition has not worsened to date (69 months after the first admission). FLAIR: fluid-attenuated inversion recovery, MRI: magnetic resonance imaging 
(a) ECD-SPECT

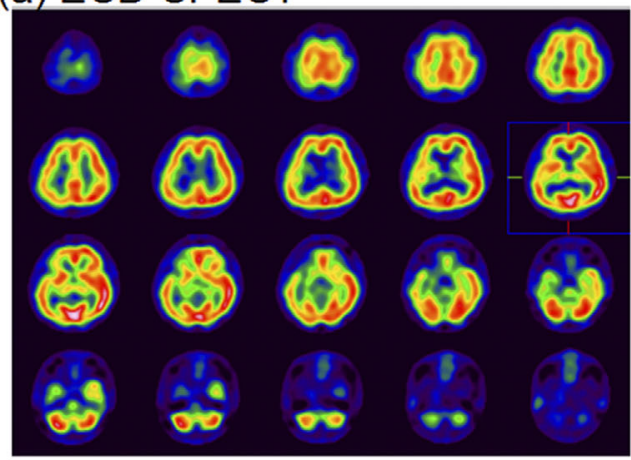

(c) IMZ-SPECT

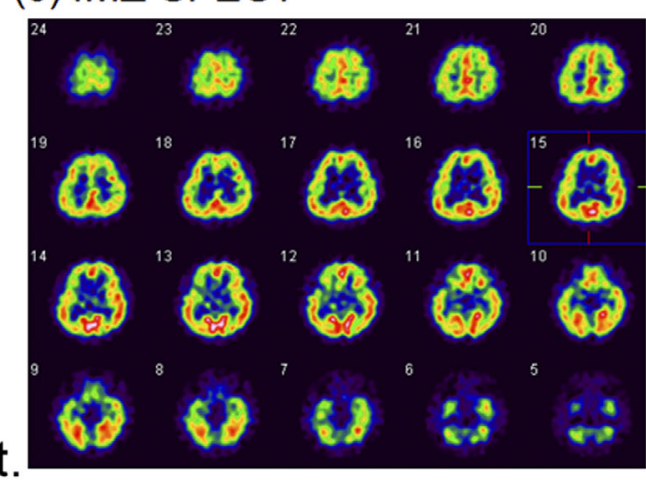

(b) ECD-SPECT

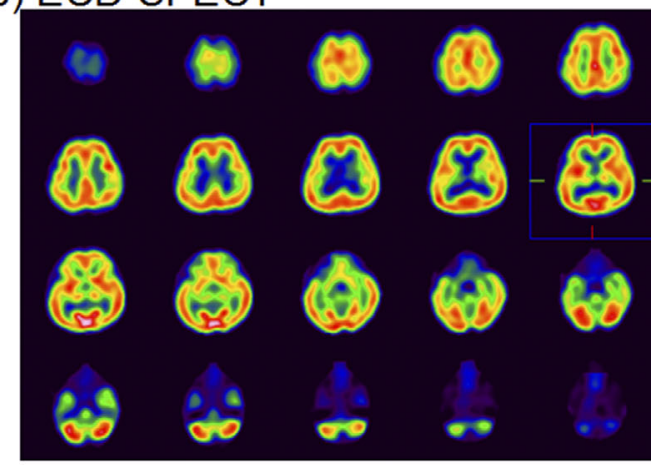

(d) IMZ-SPECT

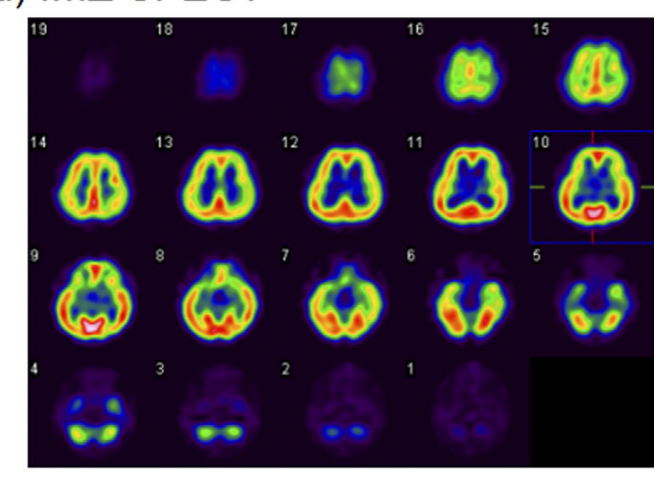

Figure 4. Follow-up ECD and IMZ-SPECT. (a and b) Follow-up ECD-SPECT revealed that the increased flow observed in the right cerebrum at the first admission had disappeared 45 (a) and 48 (b) months after the first admission. (c and d) Follow-up IMZ-SPECT showed less IMZ accumulation in the right cerebral hemisphere 45 (c) and 65 (d) months after the first admission. Note: The SPECT model used to obtain Fig. 4 was different from that used for Fig. 2 . ECD-SPECT: ${ }^{99 \mathrm{~m}}$ Tc-ethyl cysteinate dimer single-photon emission computed tomography, IMZ-SPECT: ${ }^{123}$ I-iomazenil single-photon emission computed tomography

electroencephalography revealed no epileptic discharge. The frequency of migraine attacks and imaging are shown in Fig. 6.

\section{Discussion}

We performed a detailed time-course brain imaging study of a familial hemiplegic migraine patient with a T666M mutation in the CACNA1A gene. Although a few reports have described MRI changes in the cerebrum associated with familial hemiplegic migraine, repeat imaging of our patient was performed over a four-year period, which allowed us to perform a more comprehensive evaluation of the condition. Upon his first admission to our hospital, cerebellar atrophy and a hemispheric hyperintensity signal were evident on T2weighted MRI, DWI, and FLAIR. Previous case reports also showed these findings (2-4) and indicated that the severity of these signal changes might correlate with the severity of disease symptoms. This hypothesis might be supported by the fact that the first MRI taken approximately one month before the first admission, when the patient presented with a transient mild attack, was normal.

There are two previous reports showing cerebral atrophy on MRI from one month to one year after a hemiplegic mi- graine attack $(4,5)$. As a result, cerebral atrophy may occur within a relatively short period of time. Our case also showed mild hemispheric atrophy one year after the first admission (Fig. 3c).

It is possible that this brain atrophy was due to migraineinduced ischemic changes (6). However, this speculation was not substantiated by magnetic resonance angiography performed upon admission, which revealed dilated arteries instead of stenosis, suggesting that blood flow was increased. The patient's ECD-SPECT findings also indicated increased cerebral blood flow in the right hemisphere.

In this particular case, a decreased accumulation on IMZSPECT was observed coincident with a severe migraine attack. This decline is caused by benzodiazepine receptor dysfunction because IMZ binds to benzodiazepine receptors in the cerebral cortex. IMZ-SPECT abnormalities are thought to be caused by epileptic changes (6) or neurodegeneration (7) in the cortex. In this case, decreased IMZ accumulation was not related to epilepsy because no seizure or epileptic discharge was detected by electroencephalography. It was recently reported that late IMZ-SPECT images are closely related to neurodegeneration or neuron loss $(8,9)$. We considered the brain atrophy observed in this case to be related to IMZ-SPECT abnormalities. It cannot be said that 


\begin{tabular}{|c|c|c|c|c|}
\hline \multirow{4}{*}{ frontal } & right & 52 & 45 & 51 \\
\hline \multirow{3}{*}{ temporal } & left & 42 & 50 & 54 \\
\hline \multirow{4}{*}{ parietal } & right & 55 & 41 & 47 \\
\hline \multirow{4}{*}{ cerebellum } & left & 46 & 51 & 49 \\
& right & 56 & 43 & 51 \\
\hline \multirow{3}{*}{ r/lt } & left & 39 & 49 & 50 \\
& right & 46 & 49 & 55 \\
& left & 42 & 49 & 54 \\
& & & & \\
& front & 1.24 & 0.90 & 0.94 \\
\hline & temp & 1.20 & 0.80 & 0.96 \\
\hline & pariet & 1.22 & 0.93 & 1.11 \\
\hline
\end{tabular}

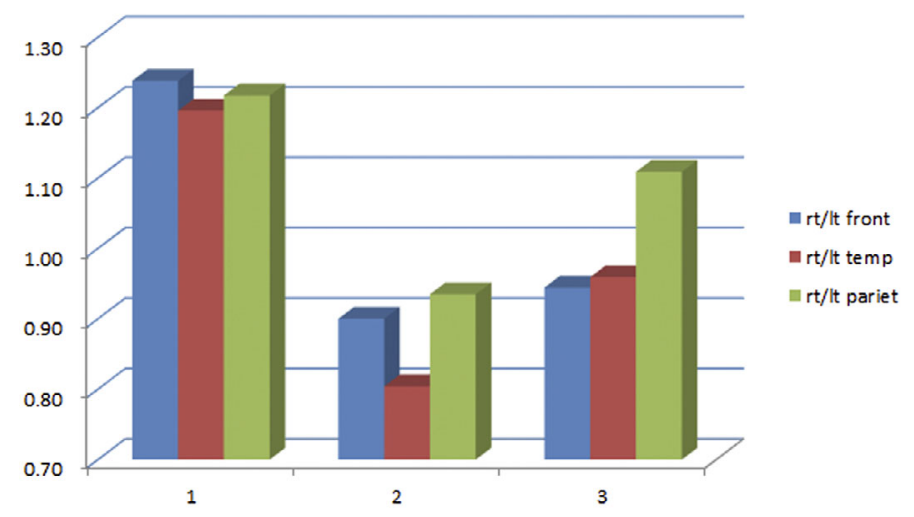

Figure 5. Regional blood flow on ECD-SPECT (Patlak Plot method). The upper table represents ECD accumulation $(\mathrm{mL} / 100 \mathrm{~g} / \mathrm{min})$ in each region and the ratio of right-: left accumulation. The lower graph also shows the ratio. During a severe migraine attack, high accumulation was observed in the right cortical hemisphere. In contrast, low accumulation was seen in the ipsilateral hemisphere 45 months later. 1: at the first admission, 2: 45 months after the first admission, 3: 48 months after the first admission. ECD-SPECT: ${ }^{99 \mathrm{~m}}$ Tc-ethyl cysteinate dimer single-photon emission computed tomography, rt: right, lt: left

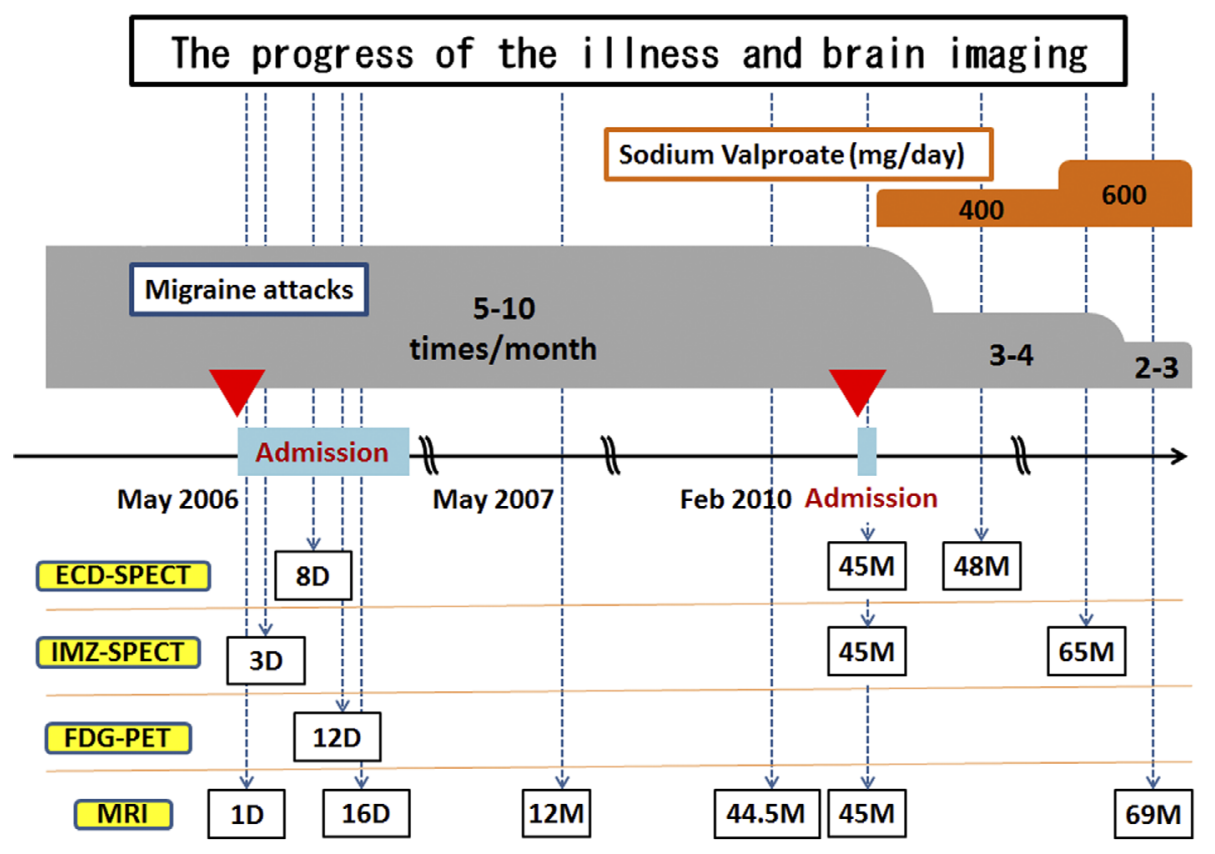

Figure 6. Illness progression and associated brain imaging. The patient was admitted to the hospital twice for severe migraine attacks with consciousness disturbance $(\nabla)$. The frequency of his migraine attacks was reduced, and severe attacks ceased after the patient began taking sodium valproate regularly. $D$ and $M$ in the squares = days and months from the first admission, respectively. ECD-SPECT: ${ }^{99 \mathrm{~m}}$ Tc-ethyl cysteinate dimer single-photon emission computed tomography, IMZSPECT: ${ }^{123}$ I-iomazenil single-photon emission computed tomography, FDG-PET: ${ }^{18}$ F-fluorodeoxyglucose-positron emission tomography 
the prevention of severe migraine attacks directly prevent brain atrophy. However, a case of brain atrophy associated with epilepsia partialis continua was reported to exhibit a decrease in IMZ accumulation (10). In addition, there was a report describing a decreased IMZ accumulation which occurred when a flow was recognized in a patient 2 months after undergoing extracranial-intracranial (EC-IC) bypass for an intracavernous aneurysm, and brain atrophy appeared 8 months later (11). The patient described herein resembles those previously reported cases in which brain atrophy occurred following a decrease in IMZ accumulation $(10,11)$.

Sensitivity to cortical spreading depression and cortical hyperexcitement was observed in knock-in mice with a CACNA1A gene mutation (12). Gutcshalk et al. reported that persistent calcium influx into excitatory synapses may disturb synaptic conduction for an extended period because FDG-PET showed hypometabolism in a hemiplegic migraine-attacked brain long after normalization, as confirmed by DWI (13). In this case, edema of the right side cerebral cortex revealed by DWI of the brain during a hemiplegic migraine suggested that dysfunction of the cerebral cortex was occurring, which was supported by a decrease in IMZ accumulation. Persistent synaptic disturbances may cause atrophic changes in the cerebral cortex. The recovery of consciousness was much faster following the patient's second admission than the first. In addition, no new changes were observed on brain MRI performed during the migraine attack. Therefore, the influence of migraine attacks at the time of the second admission might be limited.

Blood flow abnormalities and prolonged hypometabolism in a severe attack might trigger brain atrophy and a decreased IMZ accumulation. It is thus thought that preventing severe migraine attacks may reduce one of the underlying causes of brain atrophy. Therefore, it is important to suppress these migraine attacks in order to prophylactically to prevent cerebral atrophy progression. In this case, sodium valproate, which reportedly has a prophylactic effect on migraines (14), was prescribed, and the patient consented to take it after the second admission.

The authors state that they have no Conflict of Interest (COI).

\section{References}

1. Russell MB, Ducros A. Sporadic and familial hemiplegic migraine: pathophysiological mechanisms, clinical characteristics, diagnosis, and management. Lancet Neurol 10: 457-470, 2011.

2. Butteriss DJ, Ramesh V, Birchall D. Serial MRI in a case of familial hemiplegic migraine. Neuroradiology 45: 300-303, 2003.

3. Chabriat H, Vahedi K, Clark CA, et al. Decreased hemispheric water mobility in hemiplegic migraine related to mutation of CACNA1A gene. Neurology 54: 510-512, 2000.

4. Spacey SD, Vanmolkot KR, Murphy $C$, van den Maagdenberg AM, Hsiung RG. Familial hemiplegic migraine presenting as recurrent encephalopathy in a native Indian family. Headache 45: 1244-1249, 2005.

5. Hayashi R, Tachikawa H, Watanabe R, Honda M, Katsumata Y. Familial hemiplegic migraine with irreversible brain damage. Intern Med 37: 166-168, 1998.

6. Sata Y, Matsuda K, Mihara T, Aihara M, Yagi K, Yonemura Y. Quantitative analysis of benzodiazepine receptor in temporal lobe epilepsy: ${ }^{125}$ I iomazenil autoradiographic study of surgically resected specimens. Epilepsia 43: 1039-1049, 2002.

7. Ishibashi M, Sakai T, Matsuishi T, et al. Decreased benzodiazepine receptor binding in Machado-Joseph disease. J Nuclear Med 39: 1518-1520, 1998.

8. Ishihara K, Okawa M, Hishikawa T, et al. Slowly progressive neuronal death associated with postischemic hyperperfusion in cortical laminar necrosis after high-flow bypass for a carotid intracavernous aneurysm. J Neurosurg 112: 1254-1259, 2010.

9. Itoh Y, Amano T, Shimizu T, Hashimoto J, Kubo A, Fukuuchi Y. Single-Photon emission computed tomography image of benzodiazepine receptors in a patient with Creutzfeldt-Jakob disease. Intern Med 37: 896-900, 1998.

10. Kataoka H, Shinkai T, Kiriyama T, Tomomura Y, Ueno S. Benzodiazepine receptor imaging in an adult with Rasmussen's encephalitis and epilepsia partialis continua. Epileptic Disord 13: 145-149, 2011.

11. Ihara $\mathrm{K}$, Okawa M, Hishikawa $\mathrm{T}$, et al. Slowly progressive neuronal death associated with postischemic hyperperfusion in cortical laminar necrosis after high-flow bypass for a carotid intracavernous aneurysm. J Neurosurg 112: 1254-1259, 2010.

12. van den Maagdenberg AM, Pietrobon D, Pizzorusso T, et al. A Cacna1a knockin migraine mouse model with increased susceptibility to cortical spreading depression. Neuron 41: 701-710, 2004.

13. Gutcshalk A, Kollmar R, Mohr A, et al. Multimodal functional imaging of prolonged neurological deficits in a patient suffering from familial hemiplegic migraine. Neurosci Lett 332: 115-118, 2002.

14. Hering R, Kuritzky A. Sodium valproate in the prophylactic treatment of migraine: a double-blind study versus placebo. Cephalalgia 12: 81-84, 1992.

(C) 2014 The Japanese Society of Internal Medicine

http://www.naika.or.jp/imonline/index.html 